\title{
QSAR study of prolylcarboxypeptidase inhibitors by genetic algorithm: Multiple linear regressions
}

\author{
ESLAM POURBASHEER ${ }^{\mathrm{a}, *}$, SAADAT VAHDANI $^{\mathrm{b}}$, REZA AALIZADEH $^{\mathrm{c}}$, ALIREZA BANAEI $^{\mathrm{a}}$ \\ and MOHAMMAD REZA GANJALI ${ }^{\mathrm{d}, \mathrm{e}}$ \\ a'Department of Chemistry, Payame Noor University (PNU), P. O. Box, 19395-3697 Tehran, Iran \\ ${ }^{b}$ Department of Chemistry, Islamic Azad University-North Tehran Branch, Tehran, Iran \\ ${ }^{\mathrm{c}}$ Laboratory of Analytical Chemistry, Department of Chemistry, National and Kapodistrian University of \\ Athens, Panepistimiopolis Zografou, 15771 Athens, Greece \\ ${ }^{\mathrm{d}}$ Center of Excellence in Electrochemistry, Faculty of Chemistry, University of Tehran, P. O. Box \\ 14155-6455, Tehran, Iran \\ ${ }^{\mathrm{e}}$ Biosensor Research Center, Endocrinology \& Metabolism Research Center, Tehran University of Medical \\ Sciences, Tehran, Iran \\ e-mail: pourbasheer@ut.ac.ir
}

MS received 23 August 2014; revised 30 March 2015; accepted 31 March 2015

\begin{abstract}
The predictive analysis based on quantitative structure activity relationships (QSAR) on benzimidazolepyrrolidinyl amides as prolylcarboxypeptidase ( $\mathrm{PrCP})$ inhibitors was performed. Molecules were represented by chemical descriptors that encode constitutional, topological, geometrical, and electronic structure features. The hierarchical clustering method was used to classify the dataset into training and test subsets. The important descriptors were selected with the aid of the genetic algorithm method. The QSAR model was constructed, using the multiple linear regressions (MLR), and its robustness and predictability were verified by internal and external cross-validation methods. Furthermore, the calculation of the domain of applicability defines the area of reliable predictions. The root mean square errors (RMSE) of the training set and the test set for GA-MLR model were calculated to be $0.176,0.279$ and the correlation coefficients $\left(\mathrm{R}^{2}\right)$ were obtained to be $0.839,0.923$, respectively. The proposed model has good stability, robustness and predictability when verified by internal and external validation.
\end{abstract}

Keywords. QSAR; hierarchical clustering; genetic algorithms; Prolylcarboxypeptidase ( $\mathrm{PrCP})$.

\section{Introduction}

Prolylcarboxypeptidase $(\mathrm{PrCP})$ is a serine protease which has a role in metabolism regulation. A class of reversible, potent, and selective PrCP inhibitors was developed starting from a mechanism-based design for inhibiting this serine protease. ${ }^{1}$ Prolylcarboxypeptidase belongs to a class of serine peptidases that cleaves the C-terminal amino acids linked to proline in peptides such as angiotensin II (AngII), angiotensin III (AngIII) and des-Arg9-bradykinin. PrCP is widely expressed in various organs including kidney, lung, adipose, liver, brain, heart, pancreas, etc. Within these organs, this enzyme is active in both lysosomes and the membrane of human alveolar macrophages. The roles of PrCP to convert AngII to Ang 1-7 and Ang III to Ang 2-7 indicate a potential function of PrCP in both blood pressure regulation and electrolyte balance. In addition, the

*For correspondence human gene for PrCP may be related to essential hypertension. In the past several years, significant progress has been made in understanding the potential therapeutic utility of PrCP inhibition. In 2005, the Horvath group at Yale University suggested that PrCP inhibition could lead to treatment of obesity, diabetes and related conditions. Experimentally determined half maximal inhibitory concentration $\left(\mathrm{IC}_{50}\right)$ values are not always available from literature sources, and estimated values are often employed instead. Therefore, it is of interest to develop methods for estimating the PrCP inhibitory of compounds. ${ }^{2}$

Quantitative structure activity relationship (QSAR) studies provide medicinal chemists valuable information that is useful for drug design and prediction of drug activity. ${ }^{3-9}$ QSAR models are mathematical equations which construct a relationship between chemical structures and their biological activities as a linear regression model. In a typical QSAR study, one needs to find a set of molecular descriptors representing the higher 
impact on the biological activity of interest. ${ }^{10}$ Stepwise (SW) and genetic algorithm (GA) are some of the variable selection methods to build up such a set. ${ }^{11-16}$ Clearly, it is of great interest to be able to predict the activity of compounds that have no experimental values yet, as well as attemp to determine the structural parameters that the PrCP inhibition depends on. The main aim of the present work is to establish a new QSAR model for predicting prolylcarboxypeptidase (PrCP) inhibitor activities of the benzimidazolepyrrolidinyl amides using the GA-MLR technique.

\section{Computational Methodology}

\subsection{Data set}

The biological data used in this study were PrCP inhibitors, (in terms of $\mathrm{IC}_{50}$ ), of a set of 33 benzimidazolepyrrolidinyl amides. ${ }^{2}$ The activities of these inhibitors expressed by $\mathrm{IC}_{50}$ values ranging from $6.80 \mathrm{nM}$ to $8.853 \mathrm{nM}$ were converted to $\mathrm{pIC}_{50}$ (-log $\left.\left(\mathrm{IC}_{50} / \mathrm{M}\right)\right)$ values and used as the dependent variables for subsequent modeling analyses (table 1).

\subsection{Structure optimization and descriptor generation}

The 3D structures of the compounds were preoptimized using MM+ molecular mechanics force field in HyperChem program. ${ }^{17}$ The final geometries of the minimum energy conformation were obtained by a more precise optimization with the semi-empirical AM1 method. Then 1497 theoretical molecular descriptors were calculated in DRAGON program ${ }^{18}$ including (a) 0D-constitutionaldescriptors, (b) 1D-functional groups, atom centered fragments, (c) 2D-topological descriptors, walk and path counts, connectivity index, information index, various auto-correlations from the molecular graph, edge adjacency indices, descriptors of Burden eigen values, topological charge indices, eigenvalues-based indices, (d) 3D-Randic molecular profiles, geometrical descriptors, Weighted Holistic Invariant Molecular descriptors (WHIMs), Geometry, Topology and Atom-Weights Assembly (GETAWAY) descriptors, (e) charge descriptors, (f) molecular properties (calculated from models, together with some empirical descriptors). The list and meaning of the molecular descriptors can be found from the DRAGON package, and the calculation procedure is explained in detail in the Handbook of Molecular Descriptors. ${ }^{19}$ Constant and near-constant (descriptors with only one value different from the remaining ones) variables contain no useful information. In addition, to decrease the redundancy existing in the descriptors data matrix, the correlations of descriptors with each other and with pIC50 of the molecules are examined, and collinear descriptors $(\mathrm{R}<0.9)$ are detected. Those descriptors which have the pair wise correlation coefficient above 0.9 and having the lower correlation with $\mathrm{pIC}_{50}$ values are removed from the data matrix. They were excluded in the pre-reduction step and 292 descriptors were left for variable selection.

\subsection{Data splitting}

As one of the most important steps in a QSAR study is the generation of training and test sets, clustering method have been used to classify the data set. Hierarchical cluster analysis is a statistical method for finding relatively homogeneous clusters of cases based on measured characteristics. It starts with each case in a separate cluster and then combines the clusters sequentially, reducing the number of clusters at each step until only one cluster is left. When there are $\mathrm{N}$ cases, this involves N-1 clustering steps, or fusions. This hierarchical clustering process can be represented as a tree, or dendrogram, where each step in the clustering process is illustrated by a joint of the tree. The dendrogram of used data set is shown in figure 1. The training set and test set were randomly selected from the clusters while considering both the distribution of biological data and structural diversity.

\subsection{Genetic algorithm}

Genetic algorithm (GA) was used as feature selection method to search for those descriptors with minimum collinearity among each other and with significant explained variance. In GA, each individual of the population is delineated by a chromosome of binary values. In genetic terms, each variable is called a gene and set of variables is called a chromosome. It should be noted that the population of the first generation was selected randomly and the state of each variable is represented by the value of 1 (selected to being the model) or the value of zero (not selected). Thus, the genes take value of 1 or 0 . The selected variables (genes with a value of 1) were kept proportionately to collect a small subset of descriptors, which is the probability of generating 0 for a gene was set greater (at least $60 \%$ ) than the value of 1 . For different runs, the population size was varied between 50 and 200. The fitness value for each chromosome was calculated as the leave one out cross -validation correlation coefficient $\left(\mathrm{Q}_{\mathrm{LOO}}^{2}\right)^{20,21}$ 
Table 1. Experimental and predicted inhibition activity $\left(\mathrm{pIC}_{50}\right)$ of compounds by GA-MLR method.

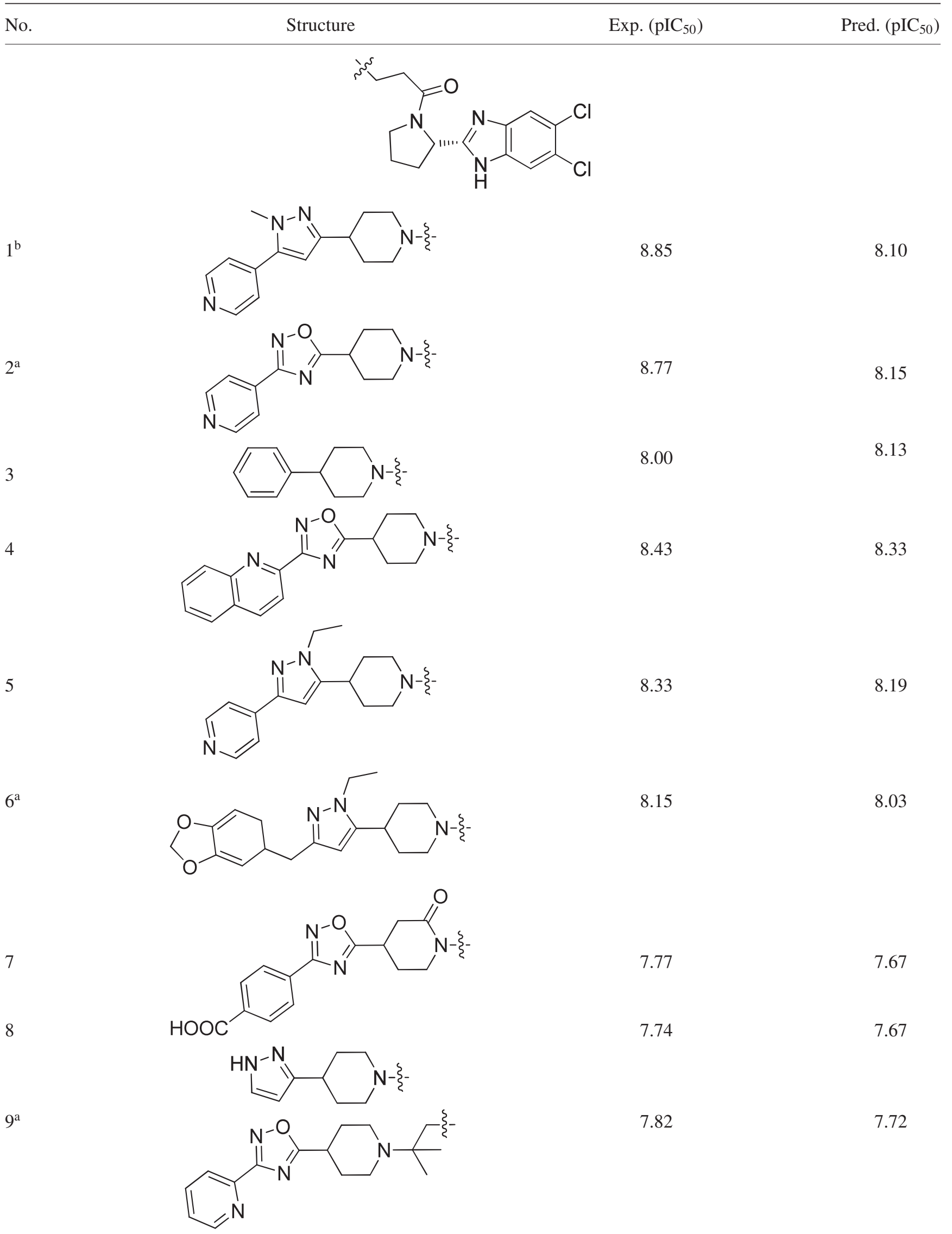


Table 1. (continued)

\begin{tabular}{|c|c|c|c|}
\hline No. & Structure & Exp. $\left(\mathrm{pIC} \mathrm{I}_{50}\right)$ & Pred. $\left(\mathrm{pIC}_{50}\right)$ \\
\hline $10^{\mathrm{a}}$ & & 7.60 & 7.48 \\
\hline 11 & & 7.92 & 7.82 \\
\hline 12 & & 7.80 & 8.06 \\
\hline 13 & & 8.07 & 8.04 \\
\hline 14 & & 7.62 & 7.54 \\
\hline $15^{\mathrm{a}}$ & & 7.68 & 7.55 \\
\hline 16 & & 7.74 & 7.96 \\
\hline 17 & & 7.89 & 7.96 \\
\hline 18 & & 7.66 & 7.41 \\
\hline 19 & & 7.72 & 7.73 \\
\hline 20 & & 7.60 & 7.49 \\
\hline
\end{tabular}


Table 1. (continued)

\begin{tabular}{|c|c|c|c|}
\hline No. & Structure & Exp. $\left(\mathrm{pIC}_{50}\right)$ & Pred. $\left(\mathrm{pIC}_{50}\right)$ \\
\hline 21 & & 8.64 & 8.33 \\
\hline 22 & & 7.57 & 7.32 \\
\hline 23 & & 7.52 & 7.67 \\
\hline 24 & & 7.49 & 7.93 \\
\hline 25 & & 7.47 & 7.43 \\
\hline $26^{\mathrm{a}}$ & & 7.38 & 7.57 \\
\hline 27 & & 7.36 & 7.32 \\
\hline 28 & & 7.19 & 7.07 \\
\hline 29 & & 7.15 & 7.08 \\
\hline 30 & & 7.03 & 7.29 \\
\hline 31 & & 6.89 & 7.02 \\
\hline $32^{\mathrm{a}}$ & & 6.85 & 7.11 \\
\hline 33 & & 6.81 & 6.98 \\
\hline
\end{tabular}

${ }^{\mathrm{a}}$ Used as test set.

${ }^{\mathrm{b}}$ Outliers.

\section{Results and Discussion}

The prediction ability of QSAR models are affected by two factors. One is the descriptors, which must carry enough information of molecular structure for the interpretation of the activity; the other one is the modeling method employed.22 There are too many descriptors from the whole available calculated data and this 


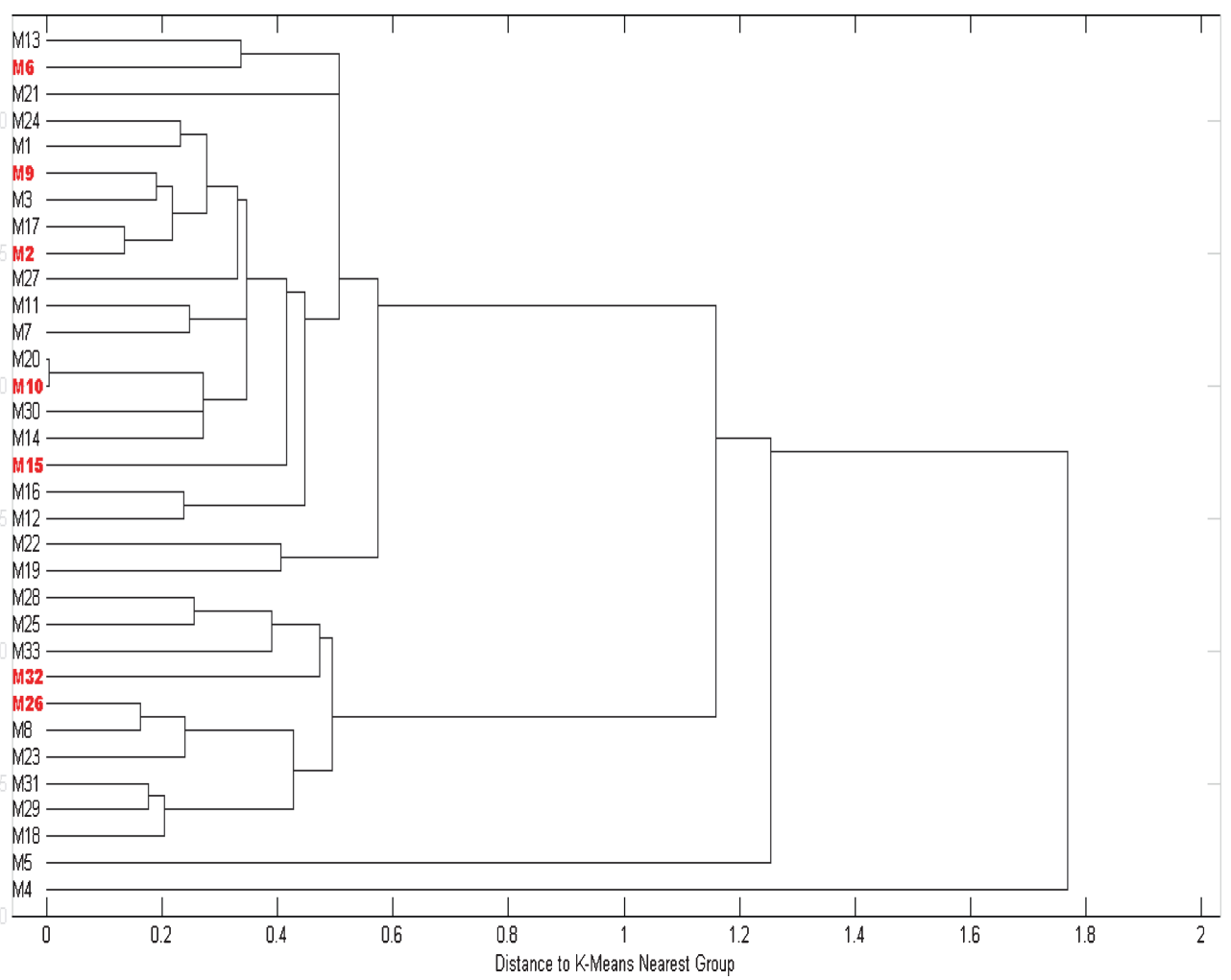

Figure 1. A dendrogram illustrating the results of the hierarchical clustering of the training and test sets.

may cause over fitting of statistical methods. Therefore, identifying important descriptors certainly plays an important role in QSAR studies. Recently, researchers have paid more attention on feature selection methodologies. Features should represent the maximum information in activity variations and collinearity among them must be kept to a minimum. In this study, five variables were selected by the genetic algorithm variable subset selection procedure. In order to build and test the model, a data set of 33 compounds was separated (by hierarchical cluster analysis) into a training set of 26 compounds, which was used to build the model and a test set of 7 compounds, which was applied to test it. With the selected five descriptors, the linear model using the training set data was built.

The correlation between prediction results and experimental values are shown in figure 2 . The square correlation coefficient $\mathrm{R}^{2}$ was obtained to be 0.772 for the training set and 0.921 for the test set with root mean square error (RMSE) of 0.234 and 0.247 , respectively. The linear equation is derived as follows:

$$
\begin{aligned}
& \mathrm{pIC}_{50}=5.799( \pm 0.3231)+1.204( \pm 0.2185) \\
& \mathrm{nR06}-2.074( \pm 0.4720) \text { Mor } 22 \mathrm{~m}+0.2710 \\
& ( \pm 0.2492) \text { Mor23e }+1.420( \pm 0.2795) \\
& \text { Mor08p }-1.250( \pm 0.3321) \text { Mor } 13 \mathrm{p}
\end{aligned}
$$

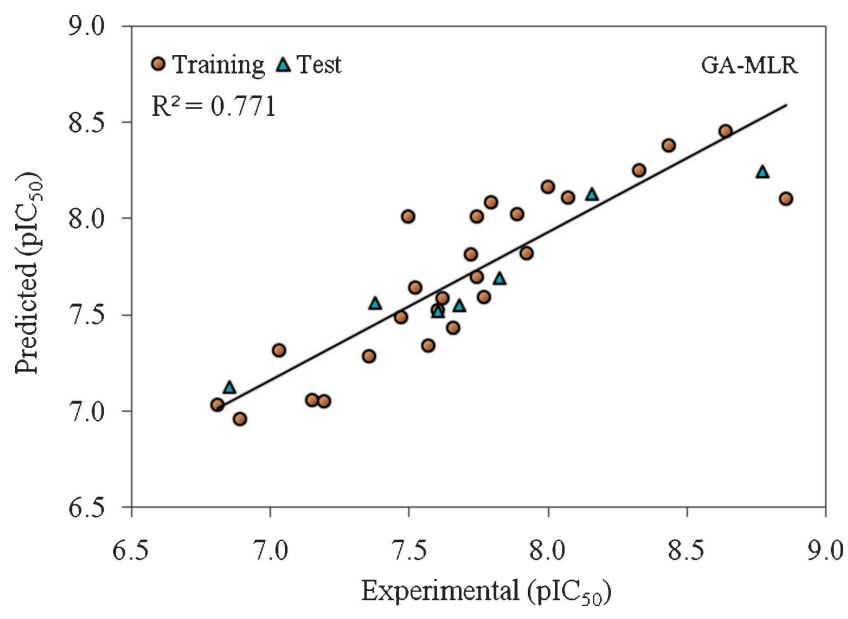

Figure 2. The predicted versus the experimental $\mathrm{pIC}_{50}$ by GA-MLR (eq. 1).

$\mathrm{R}_{\text {train }}^{2}=0.772, \mathrm{R}_{\text {test }}^{2}=0.922, \mathrm{RMSE}_{\text {train }}=0.234, \mathrm{RMSE}$ test $=0.247, \mathrm{~F}_{\text {train }}=13.508, \mathrm{~F}_{\text {test }}=0.426, \mathrm{R}_{\text {adj }}^{2}=0.714$, $\mathrm{Q}_{\mathrm{LOO}}^{2}=0.700, \mathrm{Q}_{\mathrm{LGO}}^{2}=0.655, \mathrm{Q}_{\mathrm{Boot}}^{2}=0.673, \mathrm{CCC}$ train $=0.871$, CCCtest $=0.862, \mathrm{r}^{2} \mathrm{~m}=0.628, \mathrm{Q}^{2} \mathrm{~F} 1=$ $0.806, \mathrm{Q}^{2} \mathrm{~F} 2=0.805, \mathrm{k}=1.009, \mathrm{k}^{\prime}=0.9901$

The Williams plot (figure 3), the plot of the standardized residuals versus the leverage, was exploited to 


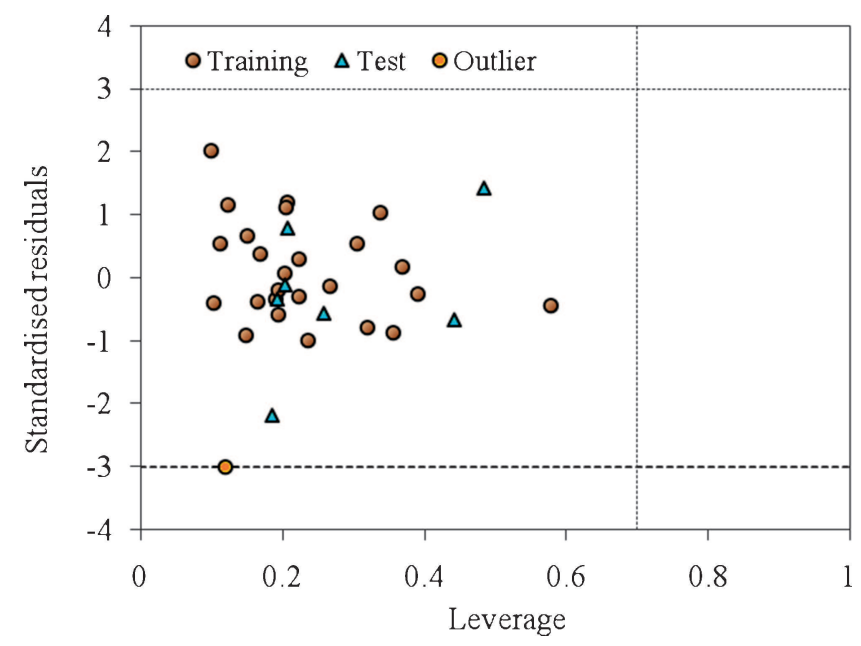

Figure 3. The William plot based on GA-MLR model (eq. 1).

visualize the applicability domain. ${ }^{23}$ The leverage of a compound in the original variable space is defined as:

$$
h_{i}=X^{T}\left(X^{T} X\right)^{-1} x_{i}
$$

where $x_{i}$ is the descriptor vector of the considered compound and $\mathrm{X}$ is the descriptor matrix derived from the training set descriptor values. The warning leverage $\left(h^{*}\right)$ is defined as: ${ }^{23}$

$$
h^{*}=\frac{3 p}{n}
$$

where $n$ is the number of calibration compounds, $p$ is the number of model variables plus one. The leverage (h) greater than the warning leverage $\left(h^{*}\right)$ suggested that the compound was very influential on the model. Moreover, a value of 3 for standardized residual is commonly used as a cut-off value for accepting predictions, because points that lie \pm 3 standardized residual from the mean cover $99 \%$ of normally distributed data. From figure 3 , it is obvious that there is only one chemical (No.1 in the training set) have the standard residuals $<3 \delta$, thus it can be regarded as structural outliers and should be remove from the data set.

After removing this compound, we have built the linear model using the training set data, and the following equation was obtained:

$$
\begin{aligned}
& \mathrm{pIC}_{50}=5.981( \pm 0.2499)+1.111( \pm 0.1678) \\
& \mathrm{nR06}-1.692( \pm 0.3714) \text { Mor22m }+ \\
& 0.3206( \pm 0.1898) \text { Mor23e }+1.341( \pm 0.2134) \\
& \text { Mor08p }-1.273( \pm 0.2525) \text { Mor13p }
\end{aligned}
$$

$\mathrm{R}_{\text {train }}^{2}=0.839, \mathrm{R}_{\text {test }}^{2}=0.923, \mathrm{RMSE}_{\text {train }}=0.176, \mathrm{RMSE}$ test $=0.279, \mathrm{~F}_{\text {train }}=19.767, \mathrm{~F}_{\text {test }}=0.292, \mathrm{R}_{\mathrm{adj}}^{2}=0.796$, $\mathrm{Q}_{\mathrm{LOO}}^{2}=0.716, \mathrm{Q}_{\mathrm{LGO}}^{2}=0.681, \mathrm{Q}_{\mathrm{Boot}}^{2}=0.710, \mathrm{CCC}$

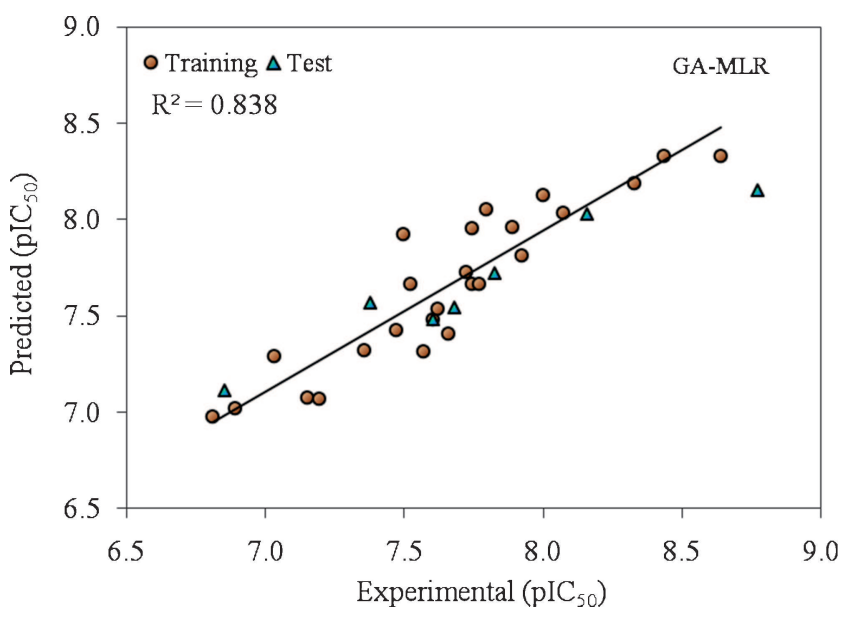

Figure 4. The predicted versus the experimental $\mathrm{pIC}_{50}$ by GA-MLR after removing outlier.

train $=0.912$, CCCtest $=0.818, \mathrm{r}^{2} \mathrm{~m}=0.578, \mathrm{Q}^{2} \mathrm{~F} 1=$ $0.758, \mathrm{Q}^{2} \mathrm{~F} 2=0.751, \mathrm{k}=1.013, \mathrm{k}^{\prime}=0.986$

In this equation, $\mathrm{N}$ is the number of compounds, $\mathrm{R}^{2}$ is the squared correlation coefficient, $\mathrm{R}_{\text {adj }}^{2}$ is adjusted $\mathrm{R}^{2}$, the $\mathrm{Q}_{\mathrm{LOO}}^{2}$ and $\mathrm{Q}_{\mathrm{LGO}}^{2}$ are the squared cross-validation coefficients for leave one out, and leave group out respectively, RMSE is the root mean square error, $r^{2} \mathrm{~m}$ is $\mathrm{r}^{2}$ modified $^{24}$ and $\mathrm{F}$ is the Fisher $\mathrm{F}$ statistic. The figures in parentheses are the standard deviations. The built model was used to predict the test set data and the prediction results are given in table 1 . The predicted values for $\mathrm{pIC}_{50}$ for the compounds in the training and test sets using equation 4 were plotted against the experimental $\mathrm{pIC}_{50}$ values in figure 4 . As can be seen from figure 4 , the calculated values for the $\mathrm{pIC}_{50}$ are in good agreement with those of the experimental values.

To measure the internal validation of this QSAR model upon inclusion/exclusion of compounds, leaveone-out (LOO) and leave-group-out cross validation techniques were performed. The correlation coefficient of $\mathrm{Q}_{\mathrm{LOO}}^{2}$ and $\mathrm{Q}_{\mathrm{LGO}}^{2}$ were 0.830 and 0.768 , respectively. The correlation matrix for selected descriptors was given in table 2. As it can be seen, there are not any high intercorrelations for chosen descriptors. Variation inflation factor is also calculated to show that the obtained model based on the selected descriptors is well established.

In order to give insight about the modeling robustness, Y-randomization test is usually performed for evaluation of chance correlation, which is obtained by shuffling the $\mathrm{Y}$ column vector (the $\mathrm{IC}_{50}$ ), while the $\mathrm{X}$ matrix is kept unaltered. Y-randomization test is a widely used technique to ensure the robustness of a QSAR model. ${ }^{25}$ In this test, the dependent variable 
Table 2. Correlation matrix for the selected descriptors with their VIF values.

\begin{tabular}{lcccccc}
\hline & nR06 & Mor22m & Mor23e & Mor08p & Mor13p & VIF \\
\hline nR06 & 1.0000 & 0.0000 & 0.0000 & 0.0000 & 0.0000 & 3.715 \\
Mor22m & 0.4368 & 1.0000 & 0.0000 & 0.0000 & 0.0000 & 1.671 \\
Mor23e & -0.6603 & -0.5982 & 1.0000 & 0.0000 & 0.0000 & 1.818 \\
Mor08p & -0.6414 & 0.0638 & 0.1039 & 1.0000 & 0.0000 & 3.273 \\
Mor13p & -0.5687 & -0.1719 & 0.1870 & 0.6607 & 1.0000 & 1.737 \\
\hline
\end{tabular}

Table 3. The $\mathrm{R}_{\text {train }}^{2}$ and $\mathrm{Q}_{\mathrm{LOO}}^{2}$ values after several $\mathrm{Y}$ randomization tests.

\begin{tabular}{lcccc}
\hline No. & $\mathrm{Q}_{\mathrm{LOO}}^{2}$ & $\mathrm{R}^{2}$ & $\mathrm{R}_{\mathrm{p}}^{2}$ & ${ }^{\mathrm{c}} \mathrm{R}_{\mathrm{p}}^{2}$ \\
\hline 1 & 0.012 & 0.147 & 0.698 & 0.762 \\
2 & 0.042 & 0.334 & 0.596 & 0.651 \\
3 & 0.114 & 0.101 & 0.720 & 0.787 \\
4 & 0.006 & 0.259 & 0.639 & 0.697 \\
5 & 0.002 & 0.197 & 0.672 & 0.734 \\
6 & 0.166 & 0.139 & 0.702 & 0.766 \\
7 & 0.093 & 0.123 & 0.710 & 0.775 \\
8 & 0.013 & 0.178 & 0.682 & 0.744 \\
9 & 0.013 & 0.295 & 0.619 & 0.675 \\
10 & 0.293 & 0.052 & 0.744 & 0.812 \\
\hline
\end{tabular}

vector is randomly shuffled and a new QSAR model is developed using the original independent-variable matrix. The processes repeated several times. If the model has low $\mathrm{R}^{2}$ and $\mathrm{Q}_{\mathrm{LOO}}^{2}$, it implies that an acceptable QSAR can be obtained for the given dataset by the current modeling method. In present study, a ten time test was developed. The $\mathrm{R}^{2}$ and $\mathrm{Q}_{\mathrm{LOO}}^{2}$ of the GAMLR model are shown in table 3. From table 3, we can see that the model has low $\mathrm{R}^{2}$ and $\mathrm{Q}_{\mathrm{LOo}}^{2}$. Moreover, ${ }^{c} R_{p}^{2}$ and $R_{p}^{2}$ values ${ }^{26}$ for Y-randomization were calculated (table 3) in which the threshold value for these parameters are 0.5 . The model exceeding this threshold is considered to be robust and not outcome of chance. The results illustrated once more that the linear MLR technique combined with a successful variable selection procedure is adequate to generate an efficient QSAR model for predicting the $\mathrm{pIC}_{50}$ value of PrCP inhibitors.

\subsection{Interpretation of descriptors}

As well as demonstrating statistical significance, QSAR model should also provide useful chemical insights into the mechanism of inhibitory activity. For this reason, an acceptable interpretation of the QSAR results is provided below. By interpreting the descriptors contained in the model, it is possible to gain some insights into factors which are related to the PrCP inhibitor activity.
First descriptor is the number of 6-membered rings (nR06) which is one of the constitutional descriptors. Constitutional descriptors are the most simple and commonly used descriptors, reflecting the molecular composition of a compound without any information about its molecular geometry. Positive coefficient of nR06 descriptor in model indicates that increase in total number of six-membered ring maybe beneficial for PrCP inhibitor activity $\left(\mathrm{pIC}_{50}\right)$.

Second descriptor is the Mor22m (3D-MoRSE - signal 22 /weighted by atomic masses). It is one of the 3D-MoRSE descriptors. 3D MoRSE descriptors (3D Molecule Representation of Structures based on Electron diffraction) are derived from Infrared spectra simulation using a generalized scattering function. The Mor22 $\mathrm{m}$ is associated with negative regression coefficient. It indicates that a decrease in the corresponding $3 \mathrm{D}-\mathrm{MoRSE}$ signal at scanning distance of 22, increase the $\mathrm{pIC}_{50}$ value.

Third descriptor is the 3D-MoRSE-signal 23/weighted by atomic Sanderson electronegativities (Mor23e). Next descriptors are the Mor08p and Mor13p. Mor08p and Mor13p were proposed as signal 08/weighted and 13/weighted by atomic polarizabilities which relates to polarizability of the molecule. Mor23e and Mor13p display a positive sign, which indicates that the $\mathrm{pIC}_{50}$ is directly related to these descriptors while Mor $13 p$ display a negative sign.

Summarizing, it is concluded that number of 6membered rings, atomic mass, atomic polarizability and atomic electronegativity play a main role in the $\mathrm{PrCP}$ inhibitor activity of studied compounds.

\section{Conclusions}

In this work, the quantitative structure- activity relationship of a series of prolylcarboxypeptidase inhibitors was carefully investigated. Various kinds of molecular descriptors were calculated to represent the molecular structures of compounds and the best-fitting descriptors were selected by using the genetic algorithm. The hierarchical clustering method was used to split the 
whole dataset into training and test set. Then a suitable model was established by GA-MLR using training data only. After discussing the physical and chemical meaning of the selected descriptors, we found out the key factors influencing the activity of benzimidazolepyrrolidinyl amides based PrCP inhibitors and provide some insights into what structural features are related to the inhibitory activity of compounds.

\section{Acknowledgements}

The authors would like to thank the State Scholarships' Foundation of Greece (I.K.Y.) for financial support.

\section{References}

1. Zhou C, Garcia-Calvo M, Pinto S, Lombardo M, Feng Z, Bender K, Pryor K D, Bhatt U R, Chabin R M, Geissler W M, Shen Z, Tong X, Zhang Z, Wong K K, Roy R S, Chapman K T, Yang L and Xiong Y 2010 J. Med. Chem. $\mathbf{5 3 7 2 5 1}$

2. Shen H C, Ding F-X, Zhou C, Xiong Y, Verras A, Chabin R M, Xu S, Tong X, Xie D, Lassman M E, Bhatt U R, Garcia-Calvo M M, Geissler W, Shen Z, Chen D, SinhaRoy R, Hale J J, Tata J R, Pinto S, Shen D-M and Colletti S L 2011 Bioorg. Med. Chem. Lett. 211299

3. Wold S, Trygg J, Berglund A and Antti H 2001 Chemom. Intell. Lab. Syst. 58131

4. Schmidli H 1997 Chemom. Intell. Lab. Sys. 37125

5. Hemmateenejad B, Miri R, Akhond M and Shamsipur M 2002 Archiv. der. Pharmazie. 335472

6. Hemmateenejad B, Miri R, Akhond M and Shamsipur M 2002 Chemom. Intell. Lab. Syst. 6491

7. Hansch C, Kurup A, Garg R and Gao H 2001 Chem. Rev. 101619
8. Hansch C, Hoekman D and Gao H 1996 Chem. Rev. 96 1045

9. Pourbasheer E, Aalizadeh R, Ganjali M, Norouzi P, Shadmanesh J and Methenitis C 2013 Med. Chem. Res. 232264

10. Pourbasheer E, Beheshti A, Khajehsharifi H, Ganjali M R and Norouzi P 2013 Med. Chem. Res. 224047

11. Sabet R and Fassihi A 2008 Int. J. Mol. Sci. 92407

12. Fassihi A and Sabet R 2008 Int. J. Mol. Sci. 91876

13. Fassihi A, Abedi D, Saghaie L, Sabet R, Fazeli H, Bostaki G, Deilami O and Sadinpour H 2009 Eur. J. Med. Chem. 442145

14. Consonni V, Todeschini R, Pavan M and Gramatica P 2002 J. Chem. Inf. Comput. Sci. 42693

15. Hansch C, Leo A and Hoekman D H, Exploring QSAR. (American Chemical Society) (1995)

16. Pourbasheer E, Aalizadeh R, Ganjali M and Norouzi P 2014 Med. Chem. Res. 2357

17. HyperChem, molecular modeling system. 7.03 edn. Hypercube, Inc., Gainesville, FL (2002)

18. Todeschini R, Consonni V, Mauri A and Pavan M, DRAGON. Software for the calculation of molecular descriptors, Talete srl, Milan, Italy (2005)

19. Todeschini R and Consonni V 2008 In Handbook of Molecular Descriptors (Wiley-VCH) p. 1

20. Habibi-Yangjeh A, Pourbasheer E and DanandehJenagharad M 2008 Monatsh. Chem. 1391423

21. Habibi-Yangjeh A, Pourbasheer E and DanandehJenagharad M 2009 Monatsh. Chem. 14015

22. Goodarzi M, Chen T and Freitas M P 2010 Chemom. Intell. Lab. Syst. 104260

23. Eriksson L J J, Worth A P, Cronin M T, McDowell R M and Gramatica P 2003 Environ. Health Perspect. 111 1361

24. Roy K, Chakraborty P, Mitra I, Ojha P K, Kar S and Das R N 2013 J. Comput. Chem. 341071

25. Xu X, Luan F, Liu H, Cheng J and Zhang X 2011 Spectrochim. Acta A Mol. Biomol. Spectrosc. 83353

26. Mitra I, Saha A and Roy K 2010 Mol. Simulat. 361067 